Article

\title{
The Italian Case of Lecco Innovation Living Lab: Stakeholders' Needs and Activities to Contribute to the Technological Innovation Process in Healthcare
}

\author{
Laura Marone *, Rossella Onofrio and Cristina Masella \\ Department of Management, Economics and Industrial Engineering, Politecnico di Milano, \\ Via Lambruschini 4/b, 20156 Milan, Italy; rossella.onofrio@polimi.it (R.O.); cristina.masella@polimi.it (C.M.) \\ * Correspondence: laura.marone@polimi.it; Tel.: +39-02-2399-3996
}

Received: 6 November 2020; Accepted: 7 December 2020; Published: 9 December 2020

check for updates

\begin{abstract}
Healthcare technological innovation is a very complex process in which different actors interact with each other, creating a large number of interconnections and synergies in the design of technological innovations. Despite the increasing number of living labs (LLs) in healthcare, building and maintaining LLs for technological innovation in healthcare is challenging. Collaboration with stakeholders remains an issue of major concern in healthcare. The purpose of this paper is to identify stakeholders' needs in building an LL in healthcare and to plan activities to foster the innovation process. The paper is based on an exploratory single case study investigating an Italian LL. Eight stakeholders' needs were identified and validated. Specific activities were identified as improving the innovation process in terms of the stakeholders' needs. The study contributes to the development of domain-specific knowledge and, as such, to the fostering of studies on and the implementation of LLs in healthcare.
\end{abstract}

Keywords: living labs; healthcare innovation; health technologies; multi-stakeholder network

\section{Introduction}

The literature on living labs (LLs) has increasingly acknowledged the importance of combining user-centred and open innovation approaches in the development of innovations and technologies.

The paradigm at the base of the concept of the LL takes its roots from two main predecessors: the Scandinavian cooperative (later translated to the participatory U.S. design) of the $60 \mathrm{~s}$ and $70 \mathrm{~s}$ and the European social experiments with Information Technologies (IT) in the 1980s. LL literature often ascribes its actual birth to Professor William Mitchell at the Massachusetts Institute of Technology (MIT) [1], acknowledging him as "the grandfather of Living Labs" [2] for his influential role in Europe. However, as highlighted by Leminen and Westerlund [3], the origins of the term can be attributed to a group of American researchers in the early 1990s who started using the terms "living lab" and "living laboratories" in their studies.

The concept of the LL evolved in subsequent years, receiving strong interest from policymakers and academics. While the number of LLs has increased, it is still difficult to identify a widely accepted definition of the LL in the literature [4-6]. The LL has been described as a research methodology $[7,8]$, an experimental environment [9,10], an innovation approach [11], an innovation network [12] and an infrastructure shared by different stakeholders [13]. As a result, widely different experiences coexist under the name "LL" [14].

Each LL is driven by different actors [9], assumes a plurality of network structures [15] or adopts different methodologies and tools [16]. LLs can be articulated as different types $[17,18]$ that cover different topics, subject specialities, experts and contexts, such as information and communications technology (ICT) innovation, urban sustainability [19-21] and campus life [4]. 
LLs have been strongly supported by the European Network of Living Labs (ENoLL), a non-profit organisation aimed at promoting the diffusion of LLs across Europe, monitoring LL best practices and disseminating LL results. More than 400 LL experiences have been recognised by the ENoLL over the years; these LLs are heterogeneous in terms of application, context and approach. Many of them are linked to funded projects and often seem to become inactive when the projects end. Of the LLs labelled as active on the list available on the ENoLL website, many concern the healthcare field or at least report "health and well-being" as one of their work domains and areas of interest. This is not surprising. In the last several decades, decision-makers, professionals and citizens have shown a burgeoning interest in developing effective approaches to managing emerging opportunities and challenges in the healthcare field. This is reflected in an overwhelming range of programs and initiatives dedicated to healthcare innovations in different contexts (hospital-based, home-based and wearable technologies).

The European concept of the LL, strongly supported and disseminated by the ENoLL, places user involvement at the centre, clearly emphasising the "user-centred" characteristic of the LL paradigm. However, other types of LL exist. Schuurman and colleagues [17] extrapolated from the literature four different types of LL: (i) the original "American" LL, (ii) the LL as an extension of testbeds, (iii) the LL as supporting context research and co-creation and (iv) the LL as focusing on collaboration and knowledge support activities. Besides the first three types, which all perfectly fit the "European stream," the fourth category occurs in parallel, focusing more on multi-stakeholder collaboration and knowledge sharing, which are cardinal for the success of LLs [22]. As a result, this category has less emphasis on the deepening of end-user involvement.

In healthcare, collaboration among stakeholders is considered a fundamental pillar in the process of conceiving and developing technological innovations. Technologies for health and healthcare, no matter how novel or mature, are indeed always embedded in complex socio-technical systems and immersed in dense networks of relations among different stakeholders in the healthcare ecosystem. In particular, to leverage the opportunities offered by new medical and digital technologies to create products and solutions that improve quality of life, different actors interact with each other to design technologies, creating a large number of interconnections and synergies in the process. Different professionals are constantly involved, and often more than one organisation is required for a single case to ensure that different perspectives are covered $[23,24]$. To add complexity, the health technology assessment discipline imposes strict rules for the healthcare community that require a multi-perspective approach and micro-dynamic collaborations. Moreover, the patient is assuming an increasingly central role in the innovation process. The literature on empowerment suggests that people will be in charge of managing their personal data on health and well-being, and patient involvement in the innovation process is commonly associated with significant benefits in terms of functionality, usability and fit with market needs [25]. In sum, all these factors make innovation in healthcare a very complex process in which healthcare organisations must cooperate with external actors, involving the end-users in a multidisciplinary approach [26].

With these considerations in mind, LLs, especially those focused on collaboration and knowledge support activities, seem to fit such a complex socio-technical system $[27,28]$, as the concept behind the LL highlights the importance of the network and aims at creating collaborative links among users, researchers, producers, and public and private organisations, emphasising the coexistence of heterogeneous actors and stakeholders [6,12,15,29,30]. Moreover, Haukipuro and colleagues [31], inspired by the findings of their study on an eHealth accelerator, proposed the LL approach as a design element in the accelerator model because of its proven usefulness in the development of new products and services in the field.

Even though LL literature in the healthcare context is increasing, there are still few studies on the subject. Among them, some scholars have attempted to identify a list of factors that can be used to build and manage an LL in healthcare [14,32-36]. Van Geenhuizen, for example, dedicated particular attention to the challenges related to the management of stakeholders' networks and multi-stakeholder cooperation $[35,36]$, and Callari and colleagues investigated the needs and requirements of the 
involvement of end-users as participants in LL initiatives [37]. This is in line with literature on LL implementation in other contexts [38].

Despite the fact that collaboration with stakeholders has been widely recognised as a key aspect of LLs, especially for the fourth type of LL, the vast majority of LL literature has focused on the user involvement and co-creation dimension of the LL approach, and only a few studies have examined stakeholders' needs. Accordingly, this paper aims at exploring an LL experience mainly focused on collaboration and knowledge sharing in healthcare to answer the following research questions (RQs):

RQ1: What are the main stakeholders' needs in building an LL (for technological innovation) and in contributing to its sustainability over time?

RQ2: How can the members of an LL experience the LL approach within their project activities in order to improve the technological innovation process?

To address these questions, the authors report on the EMPATIA@Lecco Project, a regional project funded by the Cariplo Foundation and the Lombardy Region, which involves clinical and technological centres of excellence in the field of rehabilitation that provide patients with a variety of different technologies for neurorehabilitation. Of paramount importance to the project is the establishment of a very complex collaborative network in a high-tech healthcare context: the Lecco Innovation Living Lab (LILL).

The paper is organised as follows: the second section describes the research approach and methodology used, the third section describes and discusses the key findings, and the final section describes the theoretical and practical implications of our findings.

\section{Materials and Methods}

\subsection{Research Approach}

When gaps in scientific literature exist, exploratory approaches are appropriate [39]. Given the exploratory nature of our RQs, we adopted a qualitative approach. Moreover, in the early stages of research, building theory from case studies may be the most appropriate approach [40], and the variety of terminologies used by practitioners and scholars in the area of LLs requires methods that allow deep insights rather than surface-level observations [41]. Accordingly, this research is based on a single case study and is articulated in two different phases in a longitudinal approach.

The case study selected was the LILL. The LILL was founded in Lecco (northern Italy), which is a territory that has a strong tradition in the field of rehabilitation and is home to many institutions (research centres and hospitals) that stand out as centres of excellence. The LILL's main area of interest is technological innovation focused on rehabilitation. The LILL was created as part of a project funded by the Cariplo Foundation and the Lombardy Region, and the network is composed of the following stakeholders: four departments of Politecnico di Milano, six institutes of the National Research Council (CNR), one Local Health Authority (ASST Lecco), two research hospitals (Scientific Institute for Research, Hospitalisation and Healthcare (IRCCS)), one rehabilitation centre, a national patient association (Italian Union for the Fight against Muscular Dystrophy (UILMD)) and one territorial association (Univerlecco). The primary ambition of the LILL is to formalise long-term research relationships among its members. Indeed, the LILL actors share more than ten years of collaborative research in neurorehabilitation in a long journey studded with different projects that have had a strong impact on the territory. Following Yin [39], this is the unique peculiarity that pushed the authors to select the LILL as the reference case study.

First, in phase I, we investigated the main stakeholders' needs with respect to the LL initiatives. Then, once we had identified and prioritised the needs and desires of the stakeholders, as an essential starting point for the innovation process [42], we co-designed the activities of our LL in phase II. This longitudinal approach was considered the most appropriate because it provided a more comprehensive method that allowed us to follow changes over time [43]. The overall research approach is shown in Figure 1. 


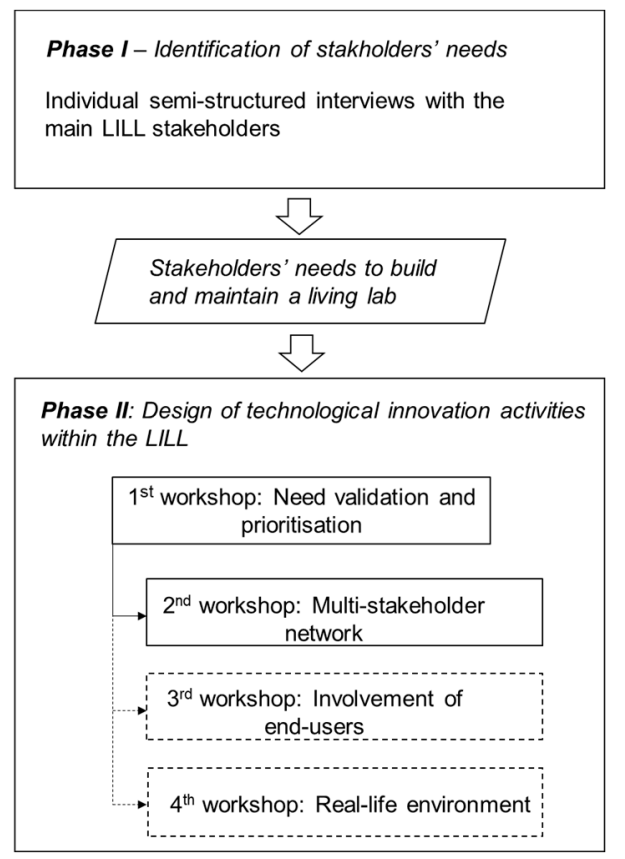

Figure 1. Study research approach. LILL: Lecco Innovation Living Lab.

\subsection{Design of Research Protocol}

In order to answer our research questions, we designed a study protocol as a guide for the entire study. It is based on the work of Leminem [6], which assumes that an LL is always formed of three main elements: (1) a multi-stakeholder network, (2) the involvement of end-users and (3) a real-life environment. Within the three elements, we identified six types of categories from the literature, as reported in Table 1.

Table 1. Living lab (LL) elements and categories.

\begin{tabular}{cc}
\hline \multicolumn{1}{c}{ LL Elements } & Categories \\
\cline { 2 - 2 } Multi-stakeholder network & Relationships among members \\
\cline { 2 - 2 } Involvement of end-users & $\begin{array}{c}\text { Management of the sharing of technology and } \\
\text { knowledge/competencies (clinical/research) }\end{array}$ \\
\cline { 2 - 2 } Real-life environment & Identification and recruitment of users (patients/citizens) \\
\hline & Definition of the function of the virtual and physical environment \\
\hline
\end{tabular}

Within the first element, the multi-stakeholder network, the identified categories are (1) relationships among members, (2) governance of the collaborative and (3) management of the sharing of technology and knowledge/competencies. This is supported by scholars who claim that differing stakeholder views and needs can lead to various difficult-to-manage conflicts [28]. Hossain and colleagues [38] suggested the importance of understanding how LL networks are organised in practice, especially in complex systems. Moreover, the importance of governance is confirmed within different contexts by Kehayia et al. [33], Van Geenhuizen [36], Kang [32] and Chrnèer [21].

Within the second element, the involvement of end-users, the following categories were identified: (1) the identification and recruitment of users and (2) the involvement of users (patients/citizens). This is supported by various papers, which have described the approaches to user involvement and 
participation in the innovation process as a key aspect of LLs [44], as presenting different ways to involve users [45] and as including their needs in every phase of the innovation process [37,46]. Many scholars have addressed the possibility of adopting this approach to accelerate innovation and knowledge enhancement through the involvement of users from the earliest stages of the process [32,36,47-49].

For the third element, a real-life environment, the category identified is the definition of the function of the virtual and physical environments. This is demonstrated by a literature debate about different sets of LL environments, which can be either (1) physical spaces, resources or virtual facilities shared among stakeholders [13] for developing innovation activities or physical representations [21] or (2) isolated spaces, such as a user's home [12], for engaging the final user in a real-life context [38].

\subsection{Data Collection and Analysis}

\subsubsection{Phase I: Identification of Stakeholders' Needs}

In the first research phase, individual semi-structured interviews were conducted to identify stakeholders' needs in building an LL and maintaining it over time. Specifically, 15 respondents were interviewed. The interviewee profiles are provided in Table 2. We gave the interviewees a brief presentation of the study and the research protocol alongside the list of the three macro LL elements and categories identified from the literature review (Table 1). For each of them, the questions attempted to capture the ways in which different LILL actors conceptualise and articulate their needs for building and maintaining LL technological innovations in healthcare over time. Each interview was audio-recorded and subsequently transcribed verbatim. The information collected was analysed using a uniform approach based on transcription, validation and integration. In particular, we used a coding technique to examine data line by line or paragraph by paragraph for significant events, experiences, feelings and so on, which were then denoted as concepts [50]. The data analysis began with first-order codes to identify the informants' views of their needs. Then, the first-order codes were organised and grouped into categories, highlighting similar shared characteristics for further investigation. The data analysis steps were continuously discussed among the authors and, when possible, with other scholars, ensuring the reliability of the analysis. Data were collected between the end of 2018 and 2019.

Table 2. Interviewees' profiles. CNR: National Research Council; IRCCS: Scientific Institute for Research, Hospitalisation and Healthcare.

\begin{tabular}{ll}
\hline & \multicolumn{1}{c}{ Interviewees' Profiles } \\
\hline 1. Internal partner representative of the CNR (IBFM: Institute of Bioimaging and Molecular Physiology) \\
2. Internal partner representative of the CNR (ICMATE: Institute of Condensed Matter Chemistry and \\
Technologies for Energy) \\
3. Internal partner representative of the CNR (IFM: Institute for Photonics and Nanotechnologies) \\
5. Internal partner representative of the CNR (INO: National Institute of Optics) \\
Internal partner representative of the CNR (STIMA: Institute of Intelligent Industrial Technologies and \\
7. Internal partner representative of the CNR (IPCB: Institute of Polymers, Composites and Biomaterials) \\
8. Internal partner representative of the Politecnico di Milano (Department of Electronics Information \\
9. Internal partner representative of the Politecnico di Milano (Department of Mechanical Engineering) \\
10. Internal partner representative of the Politecnico di Milano (Polo Lecco) \\
11. Internal clinical partner representative of the ASST Lecco \\
12. Internal clinical partner representative of the IRCCS National Institute for Elderly Rest and Care \\
13. Internal clinical partner representative of the IRCCS Medea \\
14. Internal clinical partner representative of the Villa Beretta rehabilitation centre \\
15. Board member representative of the user association
\end{tabular}


2.3.2. Phase II: Design of Technological Innovation Activities within the LILL

In the second phase, the study adopted a co-production approach by involving the relevant stakeholders in the design and delivery of the activities intended to create a successful LL approach. This phase was articulated in two workshops held between 2019 and early 2020 in Lecco (Italy). Each workshop was led by a moderator who facilitated the discussion among actors and at least one representative for each scientific/clinical partner of the project. The composition of the participants in each workshop did not change over the course of the workshops; this was done to encourage the creation of trusting relationships among the actors. Each of the co-creation workshops lasted around $120 \mathrm{~min}$; of these, around $60 \mathrm{~min}$ were dedicated to the presentation of the status of the project, the goals of the activities and the roles and responsibilities of each actor, and about $60 \mathrm{~min}$ were dedicated to discussion.

The first workshop was aimed at deepening the participants' understanding of the concept of an LL and its main components. In this workshop, a general discussion around each of the three main elements of a "traditional" LL captured in the research design was discussed in order to redefine and prioritise the key elements to be addressed regarding the LILL by gathering participants' perspectives on the topic. The second workshop took place after the end of the interviews and before launching the LILL activities. It aimed at validating the results of the interviews and addressed the co-designing of the activities to encompass the LL approach within the innovation process. This workshop was related to the first key element of this study's research framework - multi-stakeholder cooperation-as the priority for the interviewees.

The same approach will be applied to the other two key elements of our research framework, and two more co-creation workshops will be held to discuss the involvement of end-users and the real-life environment. The investigation of these two elements was put on hold due to the recent COVID-19 emergency.

\section{Results}

This section reports the results of our qualitative research about (1) stakeholders' awareness of the LL mission, (2) the identification of stakeholders' needs for the LILL's development and their contribution to its sustainability over time in a healthcare context and (3) the definition of activities that allow the participant to experience the LL approach to fostering technological innovation.

\subsection{Stakeholders' Awareness of the LL Mission}

The first category of findings concerns the level of awareness about the mission of the LILL. The interviewees stated that the LILL was born from collaborations between clinical and research actors in Lecco's geographical territory, which are mostly linked to funded research projects or gentlemen's agreements. Nevertheless, they declared that the management of the network during projects is extremely complex and unstructured. The LILL describes itself as an evolution of the existing consortium and states that its goal is to become a unique entity, facilitating and simplifying high-level interconnections to accelerate the process of innovation and the introduction of products to the market. This broad idea was interpreted and explained by our interviewees. One said, "The LILL can be considered a large box in which you can find several colours. They can all be used together or only some of them to retrieve the final product." Another interviewee also stated, "LILL can offer professionals the possibility to operate with updated infrastructures and multidisciplinary competencies. It is not only experimentation, but it is a matter of entering into a project perspective and creating together." They added, "It is an environment where everyone is free to expose themselves to others." Despite the interviewees' different points of view, they agreed on the importance of common interests in the LILL network, recognising the positive impact of receiving adequate visibility (15/15 interviewees), gaining financial benefits (15/15 interviewees), reinforcing collaborations among stakeholders and 
improving the impact of scientific output (15/15 interviewees). This unanimous agreement reflects the cultural affinity of the group that makes it extremely cohesive.

With the aim of increasing participant awareness about LLs and their key elements, the first workshop presented the different typologies of LLs, and their traditional features were discussed. Two workshop templates were proposed; they focused on the main objective of the LILL, the mission of the LILL and the three key components of LLs in general (Supplementary Materials).

All of the participants agreed in considering the LILL, under development in the EMPATIA@Lecco Project, a facilitator for multi-stakeholder collaboration and knowledge sharing following the classification proposed by Schuurman and colleagues [15]. In line with LLs in other contexts, the LILL allows the integration of both internal and external knowledge dealing with the open innovation paradigm [12,16], and it is strategic for the implementation of public, private and people partnerships (PPPPs) [51] to develop and deliver technological innovation [13]. During the workshop, all the categories proposed in the study guide were confirmed, and none of the indicated categories were defined as inappropriate or negligible. The workshop confirmed that the multi-stakeholder network dimension was the most relevant one, emphasising the role of relationships among its members, governance of the collaborative and the management of the sharing of technologies and knowledge/competencies.

The workshop also confirmed the involvement of end-users as a core aspect of the innovation process. Nevertheless, the involvement of patients from the beginning of the innovation process means dealing with stringent regulations. This is the reason why understanding how to manage the network in terms of governance and relationships between members with different institutional nature was identified by the participants as the priority for an LL based on complex networks such as these.

\subsection{Identification of Stakeholders' Needs}

Based on the interviewees' responses, the main stakeholder needs in building an LL and ensuring its sustainability over time are reported in Table 3.

Table 3. Stakeholders' needs classified according to LL elements and categories.

\begin{tabular}{|c|c|c|}
\hline LL Elements & Categories & Stakeholders' Needs \\
\hline \multirow{3}{*}{$\begin{array}{l}\text { Multi-stakeholder } \\
\text { network }\end{array}$} & Relationships among its members & $\begin{array}{l}\text { 1. Improving stakeholders' commitment and } \\
\text { building trust }\end{array}$ \\
\hline & Governance of the collaborative & $\begin{array}{l}\text { 2. Defining guidelines/rules } \\
\text { 3. Using tools and/or platforms for sharing knowledge } \\
\text { 4. Managing differences among institutional identities }\end{array}$ \\
\hline & $\begin{array}{l}\text { Managing the sharing of technology } \\
\text { and knowledge/competencies }\end{array}$ & Mapping competencies/technologies \\
\hline \multirow{2}{*}{$\begin{array}{l}\text { Involvement of } \\
\text { end-users }\end{array}$} & Identification and recruitment of users & $\begin{array}{l}\text { 6. Defining patient recruitment protocols (e.g., ensuring } \\
\text { General Data Protection Regulation (GDPR)) }\end{array}$ \\
\hline & Involvement of users & $\begin{array}{l}\text { 7. Creating different communities of patients, } \\
\text { caregivers and volunteers }\end{array}$ \\
\hline $\begin{array}{c}\text { Real-life } \\
\text { environment }\end{array}$ & $\begin{array}{l}\text { Definition of the function of virtual } \\
\text { and physical environments }\end{array}$ & $\begin{array}{l}\text { Identifying resources and infrastructures of the } \\
\text { physical space }\end{array}$ \\
\hline
\end{tabular}

The stakeholders' needs are described in more detail below.

- Improving stakeholders' commitment and building trust. 
Unstructured collaborations are strongly influenced by personal relationships, which sometimes showcases non-cooperative behaviours that negatively affect the entire innovation process. Incorrect management of interpersonal conflicts due to different backgrounds, value systems and hierarchies of roles may lead to the degradation of personal relationships. One of our respondents noted the importance of introducing the LL as a neutral and safe place, highlighting the need for trust and communication among LL actors. In addition, they said that the degree of commitment among the partners has an impact on establishing a common vision and goals. One of the interviewees said, "I realised that this type of organisation works if there is someone who strongly believes in it. Being enthusiastic is the trigger for good collaboration." Another stated, "I'm always ready to focus more on human relationships than on management schemes. Sometimes I find difficulties in interacting professionally with people with whom the human relationship is weaker." In light of this, it is clear that trust is the foundation for building and maintaining relationships over time. The interviewees further noted that improving the level of shared commitment is the key to establishing synergistic relationships and, consequently, attracting the entire network. Indeed, the stakeholders' commitment to the project and trust in one another are directly related.

- Defining guidelines/rules.

Some of the most cited issues related to network dynamics focus on how the LL is managed at both strategic and operational levels. Many respondents highlighted aspects such as access to infrastructure, conditions of use and responsibility and ownership and property rights. One of the research partners stated, "In order to organise a set of rules to manage the relationship, it is absolutely necessary to identify the spaces that will be used and the existing infrastructures to be shared, while considering issues of safety and responsibility and fees, if necessary." In addition, the clinical partners shed light on the need to define rules to manage the risk of increasing workloads and their impact on clinical routine practice. Although the workload seemed to be their major concern, the clinicians reported their willingness to share technologies and competencies for the sake of the LL.

- Using tools and/or platforms for sharing knowledge.

The aim of structuring the network is to simplify relations between stakeholders. Nevertheless, nowadays, interactions are still managed without standardised systems or infrastructures. Most interviewees considered not having a shared platform to be a barrier to overcome. One of our interviewees said, "Having a tool to manage sharing knowledge is so relevant because it is necessary to make data and information homogeneous. We are far from it. Despite the relevance of these aspects, implementing a knowledge-sharing tool or platform may not be easy, but we have to think about it."

- Managing differences among institutional identities.

Many respondents identified differences between the institutions' identities and organisational structures as a critical factor. As already mentioned, the LILL involves a highly complex network of actors, including universities, research institutes, hospitals and other clinical partners. Each of these actors has its own institutional identity. One of the clinical stakeholders explained, "Compared to research institutes, our company is a private company, so we must take a different approach because it is the owner of the clinic that makes the decisions." Considering and managing these differences between institutional identities is an essential step in building the LL and ensuring its sustainability.

- Mapping competencies/technologies.

Many interviewees reported that collaboration in such complex networks can be limited by colleagues' lack of knowledge about competencies or infrastructures. In this context, mapping enabling technologies was recognised as relevant by almost all the interviewees. In addition, the technical skills within an organisation rapidly evolve, and their continuous updating, if not monitored, was perceived 
as an obstacle. In this context, the nature of the actors involved in the network must also be considered. As mentioned previously, the LILL ecosystem is rich in clinical excellence and research in the field of rehabilitation, and in some cases, those who manage these institutes, being very proud of their work, tend to overestimate their organisational skills. These cultural aspects and continuous technological updating are aspects that must be managed.

- Defining patients' recruitment protocols (e.g., ensuring General Data Protection Regulation (GDPR)).

One particular issue for LLs in healthcare is how to involve the end-user (in this case, the patients). This is because regulation is always supervised by clinical institutions. Currently, patients can only be recruited by health professionals and only rarely can they be reached outside clinical facilities. Therefore, creating ad hoc protocols for patient involvement could be a major challenge.

- Creating different communities of patients, caregivers and volunteers.

All the interviewees agreed on the fact that the development of a network will make it easier to reach a wider audience with the innovation process. Researchers would like to involve patient associations or the specific communities of interest (elderly, children, volunteers) in different geographical areas to recruit users who, thanks to ad hoc informed consent, may participate in different projects.

- Identifying resources and infrastructures of the physical space.

According to all the interviewees, the LILL should have a physical space. The interviewees claimed that they need an emblematic space to make the network tangible, saying that "when you have a physical space, you know what you can do" and that "what you don't see may easily disappear." The place should represent the relationship among partners. As one of the research partners explained, "it gives a concrete vision to a specific objective", becoming the interface between the internal network and the external world. Therefore, its design must be considered a priority for this kind of LL.

\subsection{Structuring Activities to Experience the LL Approach}

The second workshop was mainly based on the results of the first one. The templates proposed for the discussion were focused on the first LL key element: the multi-stakeholder network. First, the stakeholders' needs identified from the interviews were validated by the workshop participants without adding any other relevant needs. In addition to the validation, the discussion revealed a clear hierarchy among the needs, with certain ones prioritised. The ranking of the needs that was created in the second workshop is as follows: first, improving stakeholders' commitment and building trust; second, defining guidelines/rules; third, mapping competencies/technologies; and fourth, using tools and/or platforms for sharing knowledge and infrastructure.

The second objective of the workshop was to co-create and plan activities to satisfy the stakeholders' needs while experimenting with the LL approach. First, to ensure the multidisciplinary nature and the involvement of the different stakeholders in each phase of the innovation process, specific working groups (WGs) were established for each technological innovation. Each WG was composed of both clinical and research partners and participated in the development of the technology throughout the whole innovation process, from conceptualisation to testing and, eventually, commercialisation. The technologies developed by each WG are listed in Table 4. Twelve of the WGs developed a novel technology, while six of them focused on developing and testing novel clinical protocols and pathways. The creation of WGs to participate in the entire innovation process was an activity that strengthened trust and knowledge sharing within the network. 
Table 4. Working groups (WGs) and the technologies developed.

\begin{tabular}{ll}
\hline WG & Technologies Developed \\
\hline 1 & Pseudo-elastic functional orthoses \\
2 & Toe-up ankle mover \\
3 & 3D-printed chest orthosis \\
4 & Variable elasticity material \\
5 & Miniature dampers \\
6 & Cellular materials for biomedical applications \\
7 & Interface materials \\
8 & Upper limb active exoskeleton \\
9 & Post-stroke assistive platform \\
10 & Rehabilitation with LINarm \\
12 & Sensor-fusion devices \\
13 & Mother-child clinical pathways \\
14 & Affective behavioural patients \\
15 & Digital environments for spatial orientation in infantile cerebral palsy \\
16 & Virtual environments for chronic respiratory diseases \\
17 & Wearable sensors \\
18 & Dementia clinical pathway \\
\hline
\end{tabular}

Second, in order to meet the needs related to managing the sharing of technology and knowledge/competencies and the governance of the collaborative, two WGs were created to work on how to share research assets. These WGs (Table 5) worked on two pilot applications focusing on 3D printers and sensors, which were considered distinctive assets for the LILL. For both research assets, a database was created as a starting point. Then, thanks to the joint work, it was possible to work on the creation of clear and standardised rules for the management of the research-sharing infrastructure. We first understood and defined the possible administrative arrangements among the LILL members, who had different institutional natures, governance styles and preferred bureaucratic procedures. We then mapped the possible service processes (linked to the shared use of these two research assets) that the LILL could offer. Finally, as the last step, standardised utilisation rules were created based on the constraints previously analysed.

Table 5. WGs and research-asset sharing.

\begin{tabular}{cc}
\hline WG & Shared Research Assets \\
\hline 1 & 3D printers belonging to the LILL members \\
2 & Wearable and customised sensors belonging to the LILL members \\
\hline
\end{tabular}

\section{Discussion and Conclusion}

In line with the complexity and the strong need for multi-stakeholder collaboration that characterises the innovation process in healthcare, this study focused on LLs as facilitators for multi-stakeholder collaboration and knowledge sharing. Beginning with a review of the literature, with a particular focus on LLs in healthcare, we found, to the best of our knowledge, no detailed analysis that considers stakeholders' needs within LLs that are mainly focused on collaboration and knowledge sharing for the technological innovation process in such a complex socio-technical system. Therefore we had two main objectives: first, we aimed to examine stakeholders' needs in building this type of LL for fostering technological innovation; second, we aimed to share how to plan specific activities to answer emerging needs. To achieve these goals, we reported on the EMPATIA@Lecco Project and adopted an empirical approach based on a single case study: the LILL.

This paper makes an initial contribution by identifying from the literature a set of key elements and categories used as a guide for the entire study (Table 1). In particular, we confirmed the concurrency of the three atomic elements of an LL [6]. Among the three key elements, the multi-stakeholder 
network dimension was revealed to be the most relevant. This finding seems to diverge from the more traditional European connotation of LLs that focuses on the involvement of the end-user. A likely reason can be found in the typology of LLs. Indeed, following the classification by Schuurman and colleagues [17], the LILL recognises itself as a "facilitator for multi-stakeholder collaboration and knowledge sharing." In this context, this finding is in line with the study by Buitendag and colleagues [22], who highlighted collaboration and knowledge support as cardinal for the success of this type of LL. Moreover, it is sustained by the particular and complex socio-technical system where the LILL is immersed in: healthcare. The dense network of relations among the different stakeholders in the innovation process and the strong need to collaborate to produce technological innovation for health both emphasise the importance of this element.

Beyond the identification of elements and categories, this study contributes by identifying a set of eight stakeholder needs (reported in Table 3) and defining an action plan to implement activities within the research and innovation processes to meet these needs. While the need identification phase concerned all elements and categories, including the involvement of end-users and the real-life environment, the second phase was limited to activities related to the multi-stakeholder network dimension as the priority for this type of LL. Two other research sessions were scheduled to investigate and co-design activities related to the remaining two elements; however, due to the COVID-19 emergency, we were forced to put these sessions on hold.

Our findings reveal that switching from a traditional research network, where the collaborations among the different actors are often informal and intermittent, to a real LL network is not always an intuitive step; we found that this transition involves a long and complex process of network and relationship structuring. In line with this consideration, our study suggests a clear hierarchy in stakeholders' needs related to the multi-stakeholder network dimension. First of all, the findings confirm the importance of "human relationships" among members, highlighting the need for commitment and trust within the network. The stakeholders clearly report the need to feel protected within the LL, which should be presented as a "neutral and safe island." This cannot disregard a careful and accurate development of rules at both operational and governance levels and an awareness of the technologies, infrastructure and skills available to the LL network. Moreover, as confirmed by our findings, when different types of stakeholders are involved, it is of paramount importance to consider and manage the different institutional natures of the actors. This is particularly true when it comes to public institutions that, at least in Italy, suffer from bureaucratic restrictions and limitations. Therefore, the actions implemented to enforce proper regulation of the LL network should inevitably pay special attention to these diversities.

In line with the literature, this specific typology of LL is predominantly long-term [17]. However, setting up an LL network able to involve prolonged engagement with stakeholders is not easy, and despite that a lot has been done to suggest and set up activities aimed at improving end-user involvement, there are still relatively few guidelines about how LL networks are organised in practice, especially in complex systems [38]. In line with this consideration, and beginning with the priority needs, we were able to contribute by identifying and designing the following activities. First, eighteen WGs were created to develop specific technological innovations. These multidisciplinary and multi-stakeholder WGs are intended to follow the innovation through the entire process, from conceptualisation to commercialisation, and their composition should remain as stable over time as possible. In this case, the team dimension is able to play an essential role in building and enforcing trusting relationships among members. Moreover, additional WGs were established and dedicated to the management and governance of shared research, competencies, infrastructures and assets among LL members, starting from two pilot applications, 3D printers and sensors, which were considered distinctive assets for the LILL. For each application, the accurate assets and competence mapping among members and the creation of specific databases helped the members promote open and shared use among the network of these assets and the related competencies. Moreover, these pilot applications helped the consortium co-create specific rules and guidelines for the collaboration that could also 
be generalised and further extended at both project and governance levels, pushing the LL network members to evaluate and choose the most suitable administrative arrangements.

In conclusion, reflecting on these findings, we suggest some practical tips that may help researchers and practitioners to switch from a traditional collaborative network to an open LL network for technological innovation in healthcare based on sharing research resources and infrastructure. First, the transition should pay particular attention to human relationships and should encourage trust among members. To this purpose, we suggest planning specific team-building activities and arranging multidisciplinary and stable WGs that follow the innovation through its entire lifecycle. In this context, beginning from well-established models in the literature, we suggest developing or adopting specific toolkits able to measure key indicators of a successful collaborative network (e.g., trust). This could help LLs make the network increasingly successful. Moreover, increasing the awareness of LL actors about the set of competencies/technologies available to LLs would help enable the effective circulation of ideas and information within a complex structure of actors. Finally, rules and guidelines to structure a "safe" and trusting relationship should include topics such as range of inclusion (especially what assets, infrastructures and technologies can be shared), access rights, safety and responsibility terms, payment and compensation policies and intellectual property rights. To support effective design and implementation of these rules and guidelines, we suggest starting from a pilot application and generalising them in an inductive approach.

\section{Limitations and Future Investigations}

Although this study contributes by fostering policies that support collaborative research in the field of technological innovation in healthcare, it suffers from some limitations.

First, the paper mainly focuses on just one of the three identified key LL elements, namely the multi-stakeholder network. As already discussed, while the first phase of the study (the stakeholders' needs identification) also included the other two elements in part (the involvement of end-users and the real-life environment), the recent COVID-19 emergency forced the activity design phase (phase II) to be limited to the multi-stakeholder network only. We are aware that an LL must incorporate all three dimensions; therefore, further research will be dedicated to investigating and planning activities for the other two LL elements (the involvement of end-users and a real-life environment).

Moreover, this study is based on a single case study and endorses a qualitative approach. A larger number of cases are necessary to enrich and improve the quality of the data and to allow for a conclusion regarding LLs in a healthcare context. Therefore, future studies need to quantitatively investigate the findings using a multiple case study approach.

Finally, since the paper suggests activities aimed at satisfying the identified needs and improving the innovation process, the satisfaction analysis of stakeholders' needs based on the activities implemented should be the next research step to enable understanding of how the analysis of practices in LLs could supplement the theory, allowing the development of models and practical methods to apply to LLs that share the same problems.

Supplementary Materials: Workshop templates, interview guide and coding template are available online at http://www.mdpi.com/2071-1050/12/24/10266/s1.

Author Contributions: Conceptualisation, C.M.; methodology, R.O.; formal analysis, R.O. and L.M.; writing-original draft preparation, L.M.; writing-review and editing, R.O. and C.M.; supervision, C.M. and R.O. All authors have read and agreed to the published version of the manuscript.

Funding: This research was funded by the Cariplo Foundation and Lombardy Region within the EMPATIA@Lecco Project (http://www.leccolivinglab.com/).

Acknowledgments: The authors express their gratitude to all the professionals involved in the LILL who shared their experiences and knowledge. The research presented in this paper originates from a development process that was carried out by continuously interacting and collaborating with all the actors of the LILL. A special acknowledgment goes to Elena Villa and Cristina De Capitani, the scientific officer of the EMPATIA@Lecco Project and the manager of Univerlecco (lead partner of the LILL), respectively. The authors gratefully acknowledge the financial support of the Lombardy Region of Italy and the Cariplo Foundation for the EMPATIA@Lecco Project. 
Conflicts of Interest: The authors declare no conflict of interest.

\section{References}

1. Pallot, M.; Trousse, B.; Senach, B.; Scapin, D. Living Lab Research Landscape: From User Centred Design and User Experience Towards User Cocreation; First European Summer School “Living Labs" Inria (ICT Usage Lab), Userlab, EsoceNet, Universcience: Paris, France, 2011.

2. Schuurman, D.; De Moor, K.; De Marez, L.; Evens, T. Living Lab research approach for mobile TV. Telemat. Inform. 2011, 28, 271-282. [CrossRef]

3. Leminen, S.; Westerlund, M. Living labs: From scattered initiatives to a global movement. Create. Innov. Manag. 2019, 28, 250-264. [CrossRef]

4. Tang, T.; Wu, Z.; Hämäläinen, M.; Ji, Y. From web 2.0 to living lab: An exploration of the evolved innovation principles. J. Emerg. Technol. Web Intell. 2012, 4, 379-385. [CrossRef]

5. Dell'Era, C.; Landoni, P. Living lab: A methodology between user-centred design and participatory design. Creat. Innov. Manag. 2014, 23, 137-154. [CrossRef]

6. Leminen, S.; Westerlund, M.; Rajahonka, M. Innovating with service robots in health. Int. J. Innov. Manag. 2017, 21, 1-24. [CrossRef]

7. Eriksson, M.; Niitamo, V.; Kulkki, S. State-of-the-Art in Utilizing Living Labs Approach to User-Centric ICT Innovation-A European Approach; Working Paper; Lulea University of Technology: Lulea, Sweden, 2005; Volume 1, pp. 1-13.

8. Dell'Era, C.D.; Landoni, P.; Gonzalez, S.J. User-centred and participatory strategies adopted by European Living Labs. Int. J. Innov. Manag. 2019, 1950048, 1-20. [CrossRef]

9. Westerlund, M.; Leminen, S. Managing the Challenges of Becoming an Open Innovation Company: Experiences from Living Labs. Technol. Innov. Manag. Rev. 2011, 1, 19-26. [CrossRef]

10. Ballon, P.; Delaer, S. Test and experimentation platforms for broadband innovation: Examining European practice. Innovation 2005, 1-22. [CrossRef]

11. Feurstein, K.; Hesmer, A.; Hribernik, K.A.S. Living Labs-A New Development Strategy. In European Living Labs-A New Approach For Human Centric Regional Innovation; Wissenschaftlicher Verlag: Berlin, Germany, 2008; pp. 1-14.

12. Nyström, A.; Leminen, S.; Westerlund, M.; Kortelainen, M. Actor roles and role patterns influencing innovation in living labs. Ind. Mark. Manag. 2014, 43, 483-495. [CrossRef]

13. Guzmán, J.G.; del Carpio, A.F.; Colomo-Palacios, R.; de Diego, M.V. Living Labs for User-Driven Innovation: A Process Reference Model. Res. Technol. Manag. 2013, 56, 29-39. [CrossRef]

14. Angelini, L.; Carrino, S.; Khaled, O.A.; Riva-Mossman, S.; Mugellini, E. Senior living lab: An ecological approach to foster social innovation in an ageing society. Future Internet 2016, 8, 50. [CrossRef]

15. Leminen, S.; Westerlund, M.; Nyström, A. Living Labs as open-innovation networks. Technol. Innov. Manag. Rev. 2012, 2, 6-11. [CrossRef]

16. Bergvall-Kåreborn, B.; Holst, M.; Ståhlbröst, A. Concept Design with a Living Lab Approach. In Proceedings of the 42nd Annual Hawaii International Conference on System Sciences, HICSS, Waikoloa, HI, USA, 5-8 January 2009; pp. 1-10. [CrossRef]

17. Schuurman, D.; Mahr, D.; De Marez, L.; Ballon, P. A Fourfold Typology of Living Labs: An Empirical Investigation amongst the ENoLL Community. In Proceedings of the International Conference on Engineering, Technology and Innovation (ICE) \& IEEE International Technology Management Conference, The Hague, The Netherlands, 24-26 June 2013.

18. Ståhlbröst, A. A set of key principles to assess the impact of Living Labs. Int. J. Prod. Dev. 2012, 17, 60-75. [CrossRef]

19. Bulkeley, H.; Coenen, L.; Frantzeskaki, N.; Hartmann, C.; Kronsell, A.; Mai, L.; Palgan, Y.V. Science Direct Urban living labs: Governing urban sustainability transitions. Curr. Opin. Environ. Sustain. 2016, $22,13-17$. [CrossRef]

20. Mulder, I.; Stappers, P.J. Co-creat ing in Practice: Results and Challenges. In Proceedings of the IEEE International Technology Management Conference (ICE), Leiden, The Netherlands, 22-24 June 2009; pp. 1-8. [CrossRef] 
21. Chronéer, D.; Ståhlbröst, A.; Habibipour, A.; Raban, J. Urban Living Labs: Towards an Integrated Understanding of their Key Components. Technol. Innov. Manag. Rev. 2019, 9, 50-63. [CrossRef]

22. Buitendag, A.A.; van der Walt, J.S.; Malebane, T.; de Jager, L. Addressing Knowledge Support Services as Part of a Living Lab Environment. Issues Inf. Sci. Inf. Technol. 2012, 9, 221-241. [CrossRef]

23. Agogué, M.; Yström, A.; Le Masson, P. Rethinking the Role of Intermediaries As an Architect of Collective Exploration and Creation of Knowledge in Open Innovation. Int. J. Innov. Manag. 2013, 17, 1350007. [CrossRef]

24. Swinkels, I.C.S.; Huygens, M.W.J.; Schoenmakers, T.M.; Nijeweme-D’Hollosy, W.O.; Velsen, L.V.; Vermeulen, J.; Schoone-Harmsen, M.; Jansen, Y.J.F.M.; Van Schayck, O.C.P.; Friele, R.; et al. Lessons learned from a living lab on the broad adoption of eHealth in primary health care. J. Med. Internet Res. 2018, 20, e83. [CrossRef]

25. Davey, S.M.; Brennan, M.; Meenana, B.J.; McAdam, R. Innovation in the medical device sector: An open business model approach for high-tech small firms. Technol. Anal. Strateg. Manag. 2011, 23, 807-824. [CrossRef]

26. Wass, S.; Vimarlund, V. Healthcare in the age of open innovation-A literature review. Health Inf. Manag. J. 2016, 45, 121-133. [CrossRef]

27. Liedtke, C.; Welfens, M.J.; Rohn, H.; Nordmann, J. Living Lab: User-Driven Innovation for Sustainability. Int. J. Sustain. High. Educ. 2012, 13, 106-118. [CrossRef]

28. Brankaert, R.; den Ouden, E. The Design-Driven Living Lab: A New Approach to Exploring Solutions to Complex Societal Challenges. Technol. Innov. Manag. Rev. 2018, 7, 44-51. [CrossRef]

29. Almirall, E.; Wareham, J. Living Labs: Arbiters of midand ground-level innovation. Technol. Anal. Strateg. Manag. 2011, 23, 87-102. [CrossRef]

30. Veeckman, C.; Schuurman, D.; Leminen, S.; Westerlund, M. Linking Living Lab Characteristics and Their Outcomes: Towards a Conceptual Framework. Technol. Innov. Manag. Rev. 2013, 6-15. [CrossRef]

31. Haukipuro, L.; Väinämö, S.; Arhippainen, L.; Ojala, T. Applying a Living Lab Approach Within an eHealth Accelerator. Technol. Innov. Manag. Rev. 2019, 9, 6-20. [CrossRef]

32. Kang, S.C. Initiation of the Suan-Lien Living Lab-A Living Lab with an Elderly Welfare Focus. Int. J. Autom. Smart Technol. 2012, 2, 189-199. [CrossRef]

33. Kehayia, E.; Swaine, B.; Longo, C.; Labbé, D.; Ahmed, S.; Archambault, P.; Poldma, T. Creating a rehabilitation living lab to optimize participation and inclusion for persons with physical disabilities. Alter 2014, 8, 151-157. [CrossRef]

34. Favela, J.; Kaye, J.; Skubic, M.; Rantz, M.; Tentori, M. Living Labs for Pervasive Healthcare Research. IEEE Pervasive Comput. 2015, 14, 86-89. [CrossRef]

35. Van Geenhuizen, M. A framework for the evaluation of living labs as boundary spanners in innovation. Environ. Plan. C Politics Space 2018, 36, 1280-1298. [CrossRef]

36. Van Geenhuizen, M. Critical factors in health innovation in cities: From ivory tower to living lab. Int. J. Glob. Environ. Issues 2014, 13, 258-280. [CrossRef]

37. Callari, T.C.; Moody, L.; Saunders, J.; Ward, G.; Holliday, N.; Woodley, J. Exploring Participation Needs and Motivational Requirements When Engaging Older Adults in an Emerging Living Lab. Technol. Innov. Manag. Rev. 2019, 9, 38-50. [CrossRef]

38. Hossain, M.; Leminen, S.; Westerlund, M. A systematic review of living lab literature. J. Clean. Prod. 2019, 213, 976-988. [CrossRef]

39. Yin, R. Case study research. In Design and Methods; Sage Publications: Thousand Oaks, CA, USA, 2009.

40. Eisenhardt, K.M. Building theories from case study research. Acad. Manag. Rev. 1989, 14, 532-550. [CrossRef]

41. Gronhaug, K.; Olson, O. Action research and knowledge creation: Merits and challenges. Qual. Mark. Res. Int. J. 1999, 2, 6-14. [CrossRef]

42. Geibler, J.; Von Piwowar, J.; Greven, A.; Brown, T. The SDG-Check: Guiding Open Innovation towards Sustainable Development Goals. Technol. Innov. Manag. Rev. 2019, 9, 20-38. [CrossRef]

43. Caruana, E.J.; Roman, M.; Hernández-Sánchez, J.; Solli, P. Longitudinal studies. J. Thorac. Dis. 2015, 7,537-540. [CrossRef]

44. Schuurman, D.; De Marez, L. Structuring User Involvement in Panel-Based Living Labs. Technol. Innov. Manag. Rev. 2012, 2, 31-38. [CrossRef]

45. Almirall, E.; Lee, M.; Wareham, J. Mapping living labs in the landscape of innovation methodologies. Technol. Innov. Manag. Rev. 2012, 2, 12-18. [CrossRef] 
46. Ståhlbröst, A. Forming Future IT: The Living Lab way of User Involvement. Ph.D. Thesis, Luleà Tekniska Universitet, Lulea, Sweden, 2008.

47. Hielkema, H.; Hongisto, P. Developing the Helsinki smart city: The role of competitions for open data applications. J. Knowl. Econ. 2013, 4, 190-204. [CrossRef]

48. Følstad, A. Living Lab for innovation development of communication technology: A literature review. Electron. J. Virtual Organ. Netw. 2008, 10, 99-131.

49. Fahy, C.; De Leon, M.P.; Stahlbrost, A.; Schaffers, H.; Hongisto, P. Services of living labs and their networks. Expand. Knowl. Econ. Issues Appl. Case Stud. 2007, 4, 713-721.

50. Corbin, J.; Strauss, A. Basics of Qualitative Research: Techniques and Procedures for Developing Grounded Theory; Sage Publications: Thousand Oaks, CA, USA, 2014.

51. Gascó, M. Living labs: Implementing open innovation in the public sector. Gov. Inf. Q. 2017, 34, 90-98. [CrossRef]

Publisher's Note: MDPI stays neutral with regard to jurisdictional claims in published maps and institutional affiliations.

(C) 2020 by the authors. Licensee MDPI, Basel, Switzerland. This article is an open access article distributed under the terms and conditions of the Creative Commons Attribution (CC BY) license (http://creativecommons.org/licenses/by/4.0/). 